\title{
Urachal Cyst, Meckel's Diverticulum and Band, and Urachus
}

\author{
Dileep Garg, ${ }^{1}$ Aditya Pratap Singh, ${ }^{2 *}$ Sunil Kothari, ${ }^{1}$ Ayush Kumar $^{2}$ \\ 1 Department of Pediatric Surgery, SN Medical College Jodhpur, Rajasthan, India. \\ 2 Department of Pediatric Surgery, SMS Medical College Jaipur, Rajasthan, India.
}

\begin{abstract}
Correspondence*: Dr. Aditya Pratap Singh, Department of Pediatric Surgery, SMS Medical College Jaipur, Rajasthan, India.
E-mail: dr.adisms@gmail.com

(C) 2017, APSP J Case Rep

Submitted: 23-07-2016

Accepted: 19-10-2016

Conflict of Interest: None

Source of Support: Nil
\end{abstract}

This is an open-access article distributed under the terms of the Creative Commons Attribution License, which permits unrestricted use, distribution, and reproduction in any medium, provided the original work is properly cited.

\section{DEAR SIR}

A 4-year old boy presented with pain abdomen, bilious vomiting and constipation for three days. On examination, abdomen was distended and tender. X-ray abdomen revealed multiple air-fluid levels suggestive of intestinal obstruction. Ultrasonography abdomen revealed dilated bowel loops with minimal free fluid in peritoneal cavity. On exploration, there was a band causing obstruction of terminal ileum which was released. Band was arising from Meckel's diverticulum which was connected to a cyst at umbilicus. On further exploration, the cyst was connected to urachus which was patent (Fig.1). There was no communication between bladder and urachus, cyst and urachus, Meckel's diverticulum and the cyst. Resection of Meckel's diverticulum with ileum and end to end ileo-ileal anastomosis was done. Urachus and cyst were also excised. Patient recovered well postoperatively. Histopathology confirmed urachal cyst, urachus, and Meckel's diverticulum.

Umbilicus is common passage for various structure during intrauterine life. These structures usually involute and their persistence result in a variety of anomalies at this site. Persistence of vitello-intestinal duct is common anomaly.[1] Urachal anomalies usually present as urachal sinus, patent urachus, or urachal cyst. Various authors have reported concurrent presence of both vitellointestinal duct and urachal anomalies presenting with various features including intestinal obstruction and persistent discharge from the umbilicus.[2-5] Our case was unique as we found a Meckel's diverticulum and a band which caused intestinal obstruction. Moreover, there was a urachal cyst at umbilicus which was attached to Meckel's diverticulum on one side and to urinary bladder on other side through a patent tract which was not communicating with the urinary bladder. This was quite unusual.

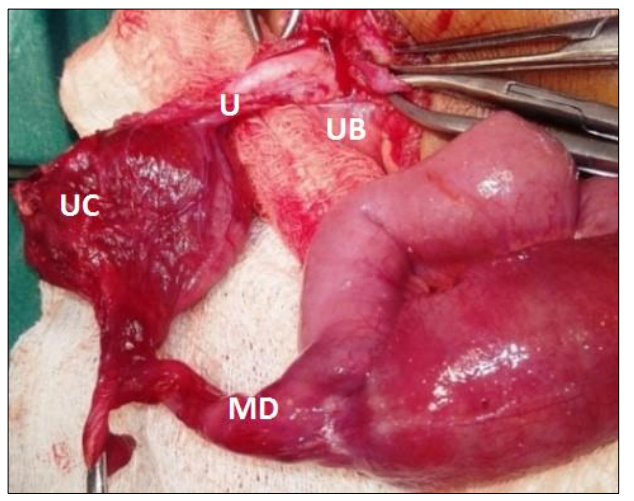

Figure 1: Showing urachal cyst (UC) attached to Meckel's diverticulum (MD) on one side and urinary bladder (UB) on other side with a patent tract- urachus $(\mathrm{U})$.

\section{REFERENCES}

1. Griffith GL, Mulcahy JJ, McRoberts JW. Umbilical abnormalities. South Med J. 1979; 72:981-4.

2. Griffith GL, Mulcahy JJ, McRoberts JW. Patent urachal associated with completely patent omphalomesenteric duct. South Med J. 1982;75:25. 
3. Nordholm-Carstensen A, Johansen LS. Intestinal obstruction caused by a Meckel's diverticulum adherent to an infected urachal cyst. Ann Pediatr Surg. 2014;10:139-41.

4. Penninga L, Timmerman P, Reurings JC, Nellensteijn DR. Simultaneous occurrence of Meckel's diverticulum and patent urachus. BMJ Case rep.2015. bcr2015210147.
5. Ozel LZ, Talu M, User Y, Aydin N, Marur T. Coexistence of a Meckel's diverticulum and a urachal remnant. Clin Anat. 2005;18:609-12. 